\title{
Erratum to: Merging scleractinian genera: the overwhelming genetic similarity between solitary Desmophyllum and colonial Lophelia
}

\author{
A. M. Addamo ${ }^{1}$, A. Vertino ${ }^{2,3}$, J. Stolarski ${ }^{4}$, R. García-Jiménez ${ }^{1}$, M. Taviani ${ }^{5,6,7}$ and A. Machordom ${ }^{\text {1* }}$
}

\section{Erratum}

As a result of vendor errors being introduced during processing, the original version of this article [1] was published with some duplication errors in Table 1.

Dd432-LpKC875348 Dd432-LpKC875349 Dd432-Lp FR821799 Dd432Dd636Dd432Dd636 Dd636LpKC87 5348Dd636LpKC875348 Dd636LpKC875348Dd636Lp KC875348 Dd636LpFR821799Dd636LpFR821799

Should be:

Dd432-LpKC875348 Dd432-LpKC875349 Dd432-Lp FR821799 Dd432-Dd636 Dd636-LpKC875348 Dd636LpKC875349 Dd636-LpFR821799

In addition, the alignment of Table 1 attribute listed as "AA" is incorrect and should correspond as shown in the below updated version.

Lastly Page 10, third paragraph included an incorrect citation. This should read as "(see Additional file 1.4, Additional file 4)" instead of "(see Additional file 1, Additional file 4)".

The original version of this manuscript has been updated to reflect the above changes.

We apologise for any inconvenience this has caused.

\section{Author details}

${ }^{1}$ Departamento de Biodiversidad y Biología Evolutiva, Museo Nacional de Ciencias Naturales (MNCN-CSIC), José Gutiérrez Abascal 2, Madrid 28006, Spain. ${ }^{2}$ Dipartimento di Scienze dell'Ambiente e del Territorio e di Scienze della Terra, Università di Milano Bicocca (UNIMIB), Piazza della Scienza 4, Milan 20126, Italy. ${ }^{3}$ Department of Geology Renard Centre of Marine Geology, Universiteit Ghent, Krijgslaan 281, Ghent B-9000, Belgium. ${ }^{4}$ Polskiej Akademii Nauk, Instytut Paleobiologii, Twarda 51/55, Warsaw PL-00-818, Poland. ${ }^{5}$ Consiglio Nazionale delle Ricerche, Istituto di Scienze Marine (ISMAR), Via Gobetti 101, Bologna 40129, Italy. ${ }^{6}$ Biology Department, Woods Hole Oceanographic Institution, 266 Woods Hole Road, Woods Hole, MA

\footnotetext{
* Correspondence: annie@mncn.csic.es

'Departamento de Biodiversidad y Biología Evolutiva, Museo Nacional de Ciencias Naturales (MNCN-CSIC), José Gutiérrez Abascal 2, Madrid 28006, Spain

Full list of author information is available at the end of the article
}

02543, USA. ${ }^{7}$ Stazione Zoologica Anton Dohrn, Villa Comunale, Naples 80121, Italy.

Received: 1 June 2016 Accepted: 8 June 2016

Published online: 19 July 2016

\section{Reference}

1. Addamo, et al. BMC Evolutionary Biology 2016;16:108. doi 10.1186/s12862-016-0654-8

\footnotetext{
Submit your next manuscript to BioMed Central and we will help you at every step:

- We accept pre-submission inquiries

- Our selector tool helps you to find the most relevant journal

- We provide round the clock customer support

- Convenient online submission

- Thorough peer review

- Inclusion in PubMed and all major indexing services

- Maximum visibility for your research
}

Submit your manuscript at www.biomedcentral.com/submit 
Table 1 Pairwise species non-synonymous substitutions with nucleotide (NT) and amino acid (AA) location

\begin{tabular}{|c|c|c|c|c|c|c|c|c|c|c|c|c|c|c|c|c|c|c|c|c|c|c|c|c|c|c|c|c|c|c|c|}
\hline \multirow[b]{2}{*}{ \# } & \multirow[b]{2}{*}{ AA } & \multirow[b]{2}{*}{ NT } & \multirow[b]{2}{*}{ Gene } & \multicolumn{4}{|c|}{ Dd432-LpKC875348 } & \multicolumn{4}{|c|}{ Dd432-LpKC875349 } & \multicolumn{4}{|c|}{ Dd432-LpFR821799 } & \multicolumn{4}{|c|}{ Dd432-Dd636 } & \multicolumn{4}{|c|}{ Dd636-LpKC875348 } & \multicolumn{4}{|c|}{ Dd636-LpKC875349 } & \multicolumn{4}{|c|}{ Dd636-LpFR821799 } \\
\hline & & & & $\mathrm{AA}$ & & & & $\mathrm{AA}$ & & & & $\mathrm{AA}$ & & & & $\mathrm{AA}$ & & & & $\mathrm{AA}$ & & & & $\mathrm{AA}$ & & & & $\mathrm{AA}$ & & & \\
\hline 1 & 26 & 77 & nad5 & 26 & R & $==>$ & K & 26 & R & $==>$ & K & 26 & R & $==>$ & K & & & & & 26 & R & $==>$ & K & 26 & R & $==>$ & K & 26 & $\mathrm{R}$ & $==>$ & K \\
\hline 2 & 38 & 113 & nad5 & 38 & T & $==>$ & 1 & 38 & T & $==>$ & 1 & 38 & T & $==>$ & 1 & 38 & T & $==>$ & 1 & & & & & & & & & & & & \\
\hline 3 & 165 & 493 & nad5 & 165 & V & $==>$ & 1 & 165 & v & $==>$ & 1 & 165 & v & $==>$ & 1 & 165 & v & $==>$ & 1 & & & & & & & & & & & & \\
\hline 4 & 351 & 1051 & nadl & & & & & & & & & & & & & 351 & K & $==>$ & Q & 351 & Q & $==>$ & K & 351 & Q & $==>$ & K & 351 & $\mathrm{Q}$ & $==>$ & K \\
\hline 5 & 427 & 1280 & nadl & 427 & c & $==>$ & $\mathrm{F}$ & 427 & C & $==>$ & $\mathrm{F}$ & & & & & 427 & $c$ & $==>$ & $\mathrm{F}$ & & & & & & & & & 427 & $\mathrm{~F}$ & $==>$ & C \\
\hline 6 & 1403 & 4208 & $\cos 3$ & & & & & & & & & & & & & 1403 & $\mathrm{~N}$ & $=\Rightarrow$ & S & 1403 & S & $=\Rightarrow$ & $\mathrm{N}$ & 1403 & S & $=\Rightarrow$ & $\mathrm{N}$ & 1403 & $S$ & $=\Rightarrow$ & $\mathrm{N}$ \\
\hline 7 & 1408 & 4225 & $\cos 3$ & 1408 & G & $==>$ & s & 1408 & G & $==>$ & s & 1408 & G & $=\Rightarrow>$ & s & 1409 & G & $==>$ & s & & & & & & & & & & & & \\
\hline 8 & 1499 & 4498 & $\cos 3$ & 1499 & S & $==>$ & G & 1499 & S & $==>$ & G & 1499 & S & $==>$ & G & & & & & 1500 & S & $==>$ & G & 1500 & S & $==>$ & G & 1500 & S & $==>$ & G \\
\hline 9 & 1685 & 5056 & $\cos 2$ & 1685 & G & $==>$ & $\mathrm{R}$ & 1685 & G & $==>$ & $\mathrm{R}$ & 1685 & G & $==>$ & $\mathrm{R}$ & 1686 & G & $=\Rightarrow>$ & R & & & & & & & & & & & & \\
\hline 10 & 2289 & 6865 & nad5 & & & & & & & & & 2288 & $\mathrm{E}$ & $==>$ & K & & & & & & & & & & & & & 2289 & $E$ & $==>$ & K \\
\hline 11 & 2471 & 7411 & $c o b$ & & & & & & & & & 2470 & $\mathrm{~T}$ & $==>$ & P & & & & & & & & & & & & & 2471 & $T$ & $==>$ & $P$ \\
\hline 12 & 2698 & 8097 & $c o b$ & 2698 & L & $==>$ & $\mathrm{F}$ & 2698 & L & $==>$ & $\mathrm{F}$ & & & & & & & & & 2699 & L & $==>$ & $\mathrm{F}$ & 2699 & L & $==>$ & $F$ & & & & \\
\hline 13 & 2863 & 8589 & nad2 & & & & & & & & & & & & & 2863 & $\mathrm{~F}$ & $==>$ & L & 2863 & L & $==>$ & $\mathrm{F}$ & 2863 & L & $==>$ & $\mathrm{F}$ & 2863 & $L$ & $==>$ & $\mathrm{F}$ \\
\hline 14 & 2930 & 8789 & nad2 & & & & & & & & & & & & & 2930 & $\mathrm{H}$ & $==>$ & $\mathrm{R}$ & 2930 & R & $==>$ & $\mathrm{H}$ & 2930 & $\mathrm{R}$ & $==>$ & $\mathrm{H}$ & 2930 & $\mathrm{R}$ & $==>$ & $\mathrm{H}$ \\
\hline 15 & 3054 & 9161 & nad2 & & & & & & & & & 3053 & L & $==>$ & S & & & & & & & & & & & & & 3054 & $L$ & $==>$ & $S$ \\
\hline 16 & 3087 & 9260 & nad6 & & & & & & & & & 3086 & L & $==>$ & P & & & & & & & & & & & & & 3087 & $\mathrm{~L}$ & $==>$ & P \\
\hline 17 & 3191 & 9574 & nad6 & 3191 & Y & $==>$ & $\mathrm{H}$ & & & & & & & & & & & & & 3192 & Y & $==>$ & $\mathrm{H}$ & & & & & & & & \\
\hline 18 & 3221 & 9664 & nad6 & 3221 & L & $==>$ & 1 & 3221 & L & $==>$ & 1 & 3221 & L & $==>$ & 1 & & & & & 3222 & L & $=\Rightarrow$ & 1 & 3222 & L & $==>$ & 1 & 3222 & $\mathrm{~L}$ & $==>$ & 1 \\
\hline 19 & 3348 & 10046 & $\operatorname{cox} 1$ & 3348 & A & $==>$ & V & 3348 & A & $==>$ & V & 3348 & A & $==>$ & V & & & & & 3349 & A & $==>$ & V & 3349 & A & $==>$ & V & 3349 & A & $==>$ & V \\
\hline 20 & 3354 & 10064 & $\operatorname{cox} 1$ & 3354 & V & $=\Rightarrow>$ & A & 3354 & V & $==>$ & A & 3354 & V & $=\Rightarrow$ & A & & & & & 3355 & v & $=\Rightarrow$ & A & 3355 & V & $==>$ & A & 3355 & V & $=\Rightarrow$ & A \\
\hline 21 & 3389 & 10166 & $\operatorname{cox} 1$ & & & & & & & & & 3388 & s & $==>$ & $\mathrm{F}$ & & & & & & & & & & & & & 3389 & S & $==>$ & $\mathrm{F}$ \\
\hline 22 & 3713 & 11141 & $\operatorname{cox} 1$ & 3713 & $\mathrm{R}$ & $=\Rightarrow$ & K & 3713 & $\mathrm{R}$ & $==>$ & K & 3713 & $\mathrm{R}$ & $=\Rightarrow$ & K & & & & & 3714 & $\mathrm{R}$ & $=\Rightarrow$ & K & 3714 & $\mathrm{R}$ & $=\Rightarrow$ & K & & & & \\
\hline
\end{tabular}

